\title{
Preconditioning Large Integral-Equation Problems Involving Complex Targets ${ }^{\dagger}$
}

\author{
Tahir Malas ${ }^{1,2}$ and Levent Gürel ${ }^{1,2 *}$ \\ ${ }^{1}$ Department of Electrical and Electronics Engineering \\ ${ }^{2}$ Computational Electromagnetics Research Center (BiLCEM) \\ Bilkent University, TR-06800, Bilkent, Ankara, Turkey \\ E-mail: tmalas@ee.bilkent.edu.tr, lgurel@bilkent.edu.tr
}

\section{Introduction}

Various real-life applications, such as radar cross section (RCS) computations of arbitrarily shaped three-dimensional (3-D) targets and complicated antenna design calculations, benefit from the accurate solutions of surface integral-equation formulations. Even though integral-equation methods yield dense matrix systems, with the multilevel fast multipole algorithm (MLFMA), the matrix-vector multiplication $(\mathrm{MVM})$ can be performed in $\mathcal{O}\left(N N_{L}\right)$ time using $\mathcal{O}\left(N N_{L}\right)$ memory, where $N_{L}$ is the number of MLFMA levels [1]. Furthermore, MLFMA has been successfully parallelized so that it scales well up to a moderate number of processors [2]. Therefore, provided that the number of iterations is not too high, it is possible to obtain efficient and accurate solutions of many real-life problems with low-cost parallel computing platforms.

For perfect electric conductor objects involving open surfaces, the electric-field integral equation (EFIE) is the inevitable choice among various integral-equation formulations. The matrices that result from EFIE are symmetric, complex, but not Hermitian. Even though the conjugate gradients (CG) method can be used with such systems, it lacks its optimal behavior and requires symmetric preconditioners; this may be a serious limitation. On the other hand, the combined-field integral equation (CFIE) is usually preferred for closed-surface problems due to its favorable convergence properties when solved with iterative methods. CFIE yields non-Hermitian systems, hence nonsymmetric solvers, such as GMRES and BiCGStab [3], are used as iterative solvers.

When the target object is large in terms of the operating wavelength, the resulting matrix equations can have millions of unknowns. From the preconditioning perspective, solutions of such problems can be challenging because of the following reasons:

- Condition numbers of the matrices can grow very large as the number of unknowns increase. This not only the case for EFIE, but also for CFIE when it is applied to complex $3-\mathrm{D}$ targets.

- Solutions of large problems put more pressure on the memory use. On the other hand, increasing condition numbers requires more robust methods, such

\footnotetext{
${ }^{\dagger}$ This work was supported by by the Scientific and Technical Research Council of Turkey (TUBITAK) under Research Grants 105E172 and 107E136, the Turkish Academy of Sciences in the framework of the Young Scientist Award Program (LG/TUBA-GEBIP/2002-1-12), and by contracts from ASELSAN and SSM.
} 
as denser preconditioners and optimal solvers, whose advantages also come with extra memory costs.

- Most of the existing preconditioners are generated from the near-field matrices, with the assumption that it is a good approximation to the dense system matrix. On the other hand, near-field matrices become sparser as problem sizes increase, and this assumption becomes questionable. Actually, in many cases, we may need more than the near-field matrix for effective preconditioning.

In this paper, we discuss the issues that we face when solving very large integralequation problems. We have previously reported three effective preconditioning techniques, i.e., a sparse approximate inverse (SAI) preconditioner, the iterative near-field (INF) preconditioner, and the approximate MLFMA (AMLFMA) preconditioner [4]. The SAI preconditioner is a low-cost, but effective preconditioner generated from the near-field matrix. The INF and AMLFMA preconditioners are more effective preconditioners that iteratively solve linear systems for preconditioning. For these preconditioners, we choose GMRES as the "inner solver" since we solve the inner systems roughly and GMRES provides rapid decrease in the residual error during the first few iterations. Since the preconditioning operation is not fixed with these preconditioners, they have to utilize a flexible solver as the "outer solver" [5]. As its name suggests, the INF preconditioner solves the near-field system for preconditioning. The AMLFMA preconditioner, on the other hand, uses a cheap version of MLFMA for MVM, hence uses more than what is provided by the nearfield interactions. We have already reported the solution of a 22-million-unknown patch problem and verified its accuracy by comparing the MLFMA solution with a physical-optics solution [4]. In the next section, we will provide the solutions of more complex and real-life problems, and discuss the difficulties that arise when the sizes of matrix equations reach millions of unknowns.

\section{Numerical Results}

For the problems reported in this section, solutions are carried out on 32 cores of an Intel Xeon cluster connected via an Infiniband network. We use GMRES and FGMRES as the solvers. We note that FGMRES stores two vectors per iteration, hence its memory cost is twice of that of GMRES. We set the the initial guess to zero and the stopping criterion as six orders of relative decay in the initial residual norm or a maximum of 1,000 iterations, unless stated otherwise. The stopping criteria of inner solvers are set to one order of relative decay or a maximum of five and ten iterations for the INF and AMLFMA preconditioners, respectively. We can achieve strong preconditioners with such loose stopping criteria, because the inner solvers of INF and AMLFMA are accelerated by SAI in a nested manner.

In Fig. 1(a), we show the solutions of a parabolic reflector using SAI, INF, and AMLFMA preconditioners up to 12 million unknowns. Without a preconditioner, the smallest problem involving 47,870 unknowns can be solved in 795 iterations with no-restart GMRES, but larger problems cannot be solved. On the other hand, SAI succeeds to solve all sizes of this problem. The INF and AMLFMA preconditioners improve the performance around $25 \%$ and $45 \%$ with respect to SAI. Moreover, 
when SAI is used with other non-optimal solvers, such as BiCGStab, the required number of MVMs grows up much faster, and it becomes too costly to solve large problems. Hence, we also show the GMRES memory cost for these preconditioners in Fig. 1(b). In addition to providing smallest solution times, by decreasing the outer iteration counts dramatically, the AMLFMA preconditioner consumes much less memory compared to the other two preconditioners.

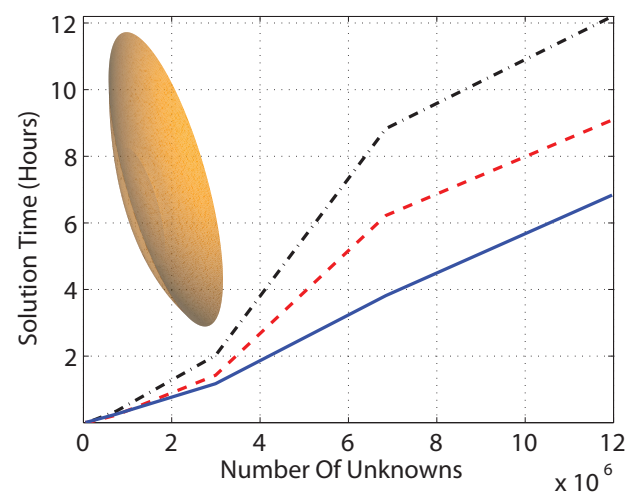

(a)

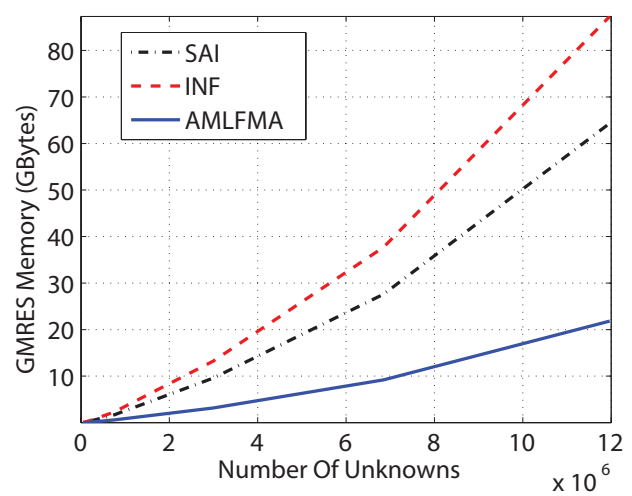

(b)

Figure 1: Comparison of SAI, INF, and AMLFMA preconditioners for the parabolic reflector with respect to (a) solution times and (b) total GMRES memory.

Next, we analyze the solution of the almond geometry, which is a closed-surface problem that can be formulated with CFIE. Instead of the INF preconditioner, we use a block-diagonal preconditioner (BDP), which is commonly used for CFIE. From Fig. 2, we realize that the solution of large closed-surface problems can also be very difficult and necessitate strong preconditioners. In particular, for the solution of the 2,140,626-unknown almond problem, BDP cannot decrease the relative residual to $10^{-3}$ in 2,000 iterations. On the other hand, SAI and AMLFMA solve this problem in 810 and 142 iterations, respectively.

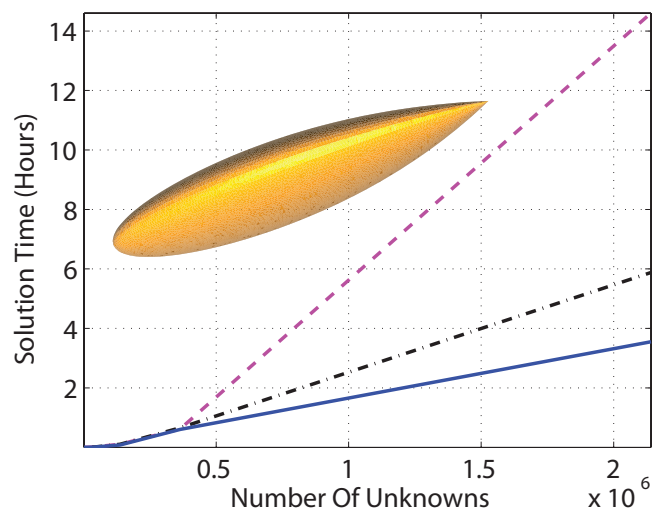

(a)

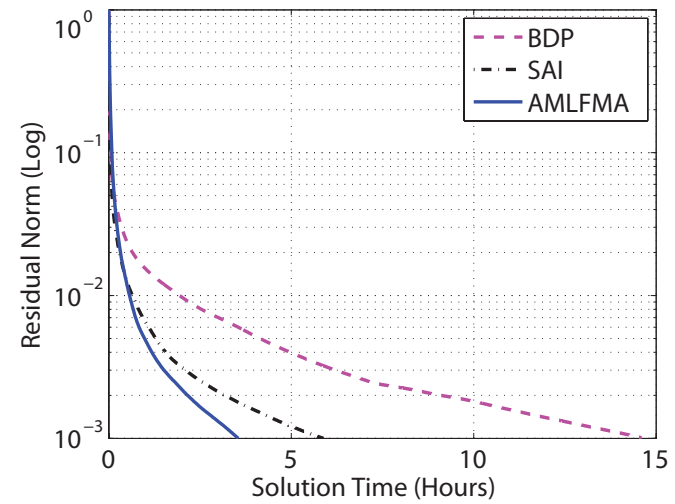

(b)

Figure 2: (a) Comparison of BDP, SAI, and AMLFMA preconditioners for the almond geometry with increasing number of unknowns. (b) The residual plot for the 2,140,626-unknown problem. 
Finally, we solve a real-life problem, i.e., a helicopter, which has a complicated geometry with many details. Fig. 3 shows the solution times up to 5.2 millions of unknowns. The use of the strong AMLFMA preconditioner helps decrease the solution times more than two-fold for this problem. The largest helicopter problem involves 5.2 million unknowns. With the AMLFMA preconditioner, including the ten-minute SAI setup time, the solution is obtained only in 46 minutes for $10^{-3}$ residual error, which is usually sufficient for accurate RCS results in practice.

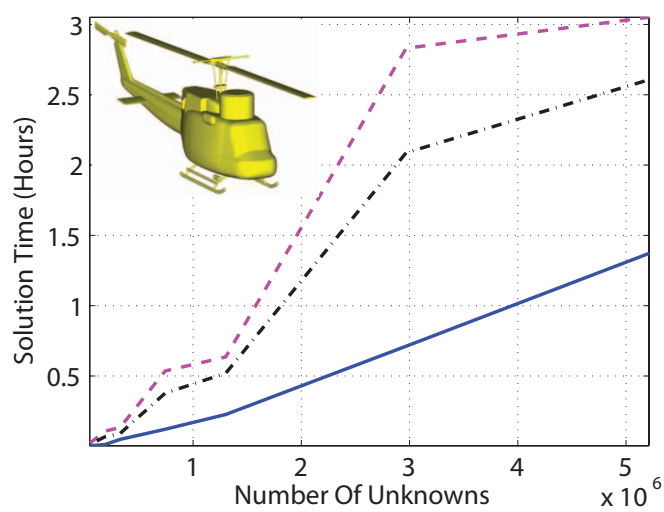

(a)

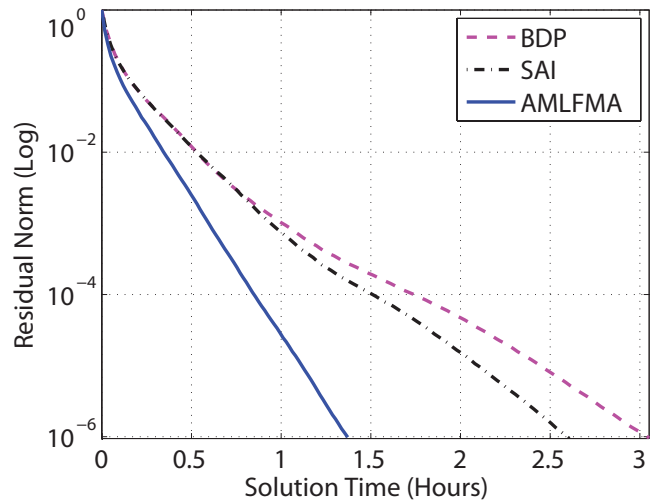

(b)

Figure 3: (a) Comparison of BDP, SAI, and AMLFMA preconditioners for the helicopter problem with increasing number of unknowns. (b) The residual plot for the 5,210,640-unknown problem.

\section{Conclusion}

When the target problem is small in terms of the wavelength, simple preconditioners, such as BDP, may sufficiently accelerate the convergence. On the other hand, for large-scale problems, the matrix equations become much more difficult to solve, and therefore, the importance of preconditioning become more evident for both formulations. In this paper, we demonstrate the use of novel, strong, and efficient preconditioners for the solutions of large EFIE and CFIE problems.

\section{References}

[1] W. C. Chew, J.-M. Jin, E. Michielssen, and J. Song, Fast and Efficient Algorithms in Computational Electromagnetics. Boston, MA: Artech House, 2001.

[2] Ö. Ergül and L. Gürel, "Efficient Parallelization of the Multilevel Fast Multipole Algorithm for the Solution of Large-Scale Scattering Problems," IEEE Trans. Antennas Propagat., submitted for publication. 2007.

[3] L. N. Trefethen and D. Bau, III, Numerical Linear Algebra. SIAM, Philadelphia, USA, 1997.

[4] T. Malas and L. Gürel, "Sequential and Parallel Preconditioners for Large-Scale Integral-Equation Problems," CEM'07 Computational Electromagnetics Workshop, pp. 35-43, Izmir, Turkey, Aug. 2007.

[5] S. Balay, W. D. Gropp, L. C. McInnes, and B. F. Smith, "PETSc Users Manual," Tech. Report ANL-95/11 - Revision 2.1.5, Argonne National Laboratory, 2004. 